\title{
Tuning of the two electron states in quantum rings through the spin-orbit interaction
}

\author{
Y. Liu, ${ }^{1}$ F. Cheng, ${ }^{1}$ X. J. Li, ${ }^{2}$ F. M. Peeters, ${ }^{3}$ and Kai Chang ${ }^{1, *}$ \\ ${ }^{1}$ SKLSM, Institute of Semiconductors, Chinese Academy of Sciences, P.O. Box 912, Beijing 100083, China \\ ${ }^{2}$ School of Physics and Optoelectronics Technology, Fujian Normal University, Fuzhou 350007, China \\ ${ }^{3}$ Department of Physics, University of Antwerp, Groenenborgerlaan 171, B-2020 Antwerpen, Belgium
}

(Received 24 February 2010; revised manuscript received 28 May 2010; published 22 July 2010)

\begin{abstract}
The effect of the Coulomb interaction on the energy spectrum and anisotropic distribution of two electron states in a quantum ring in the presence of Rashba spin-orbit interaction (RSOI) and Dresselhaus SOI (DSOI) is investigated in the presence of a perpendicular magnetic field. We find that the interplay between the RSOI and DSOI makes the single quantum ring behaves like a laterally coupled quantum dot and the interdot coupling can be tuned by changing the strengths of the SOIs. The interplay can lead to singlet-triplet state mixing and anticrossing behavior when the singlet and triplet states meet with increasing magnetic field. The two electron ground state displays a bar-bell-like spatial anisotropic distribution in a quantum ring at a specific crystallographic direction, i.e., [110] or [1 $\overline{1} 0]$, which can be switched by reversing the direction of the perpendicular electric field. The ground state exhibits a singlet-triplet state transition with increasing magnetic field and strengths of RSOI and DSOI. An anisotropic electron distribution is predicted which can be detected through the measurement of its optical properties.
\end{abstract}

DOI: $10.1103 /$ PhysRevB.82.045312

PACS number(s): 73.21.La, 71.70.Ej, 71.35.Cc

\section{INTRODUCTION}

All-electrical control of spin states is an important issue in spintronics and quantum information processing. ${ }^{1-4}$ The electrically tunable spin-orbit interaction ${ }^{5-8}$ (SOI) provides us an efficient way to control spin which has become one of the most influential concepts in semiconductor spintronics. ${ }^{9-11}$ An electron moving in an electric field feels an effective magnetic field $\vec{B}_{\text {eff }} \propto \vec{E} \times \vec{p}$, where $\vec{E}$ is an external electric field and $\vec{p}$ is the electron momentum. This effective magnetic field interacts with the electron spin and lifts the electron spin degeneracy. The asymmetry can arise from structural inversion asymmetry of the confining potential for electrons, called the Rashba spin-orbit interaction (RSOI) (Refs. 12 and 13) and from bulk inversion asymmetry, i.e., the Dresselhaus SOI (DSOI). ${ }^{14}$ The strength of the RSOI can be tuned by an external gate voltage or by asymmetric doping. ${ }^{5,6,15-17}$ In thin quantum wells, the strength of the DSOI is comparable to that of the RSOI since the strength of the DSOI depends heavily on the thickness of the quantum well ( $\propto 1 / L_{z}^{2}$, where $L_{z}$ is the thickness of the quantum well). ${ }^{10}$ The SOIs behave like an in-plane magnetic field and the direction of the total effective in-plane magnetic field varies with the in-plane momentum, which is the dominant spin relaxation mechanism in a semiconductor twodimensional electron gas. ${ }^{18-21}$

Quantum rings, artificial atoms, or molecules with unique topological geometry, have attracted a lot of attention due to the appearance of several interesting phenomena, e.g., the Aharonov-Bohm (AB)-type and the Aharonov-Casher (AC)type effects and magnetic field driven transitions of the multielectron ground state. ${ }^{7,22-24}$ These interesting physics arises from the interplay between the Coulomb interaction, the unique topological geometry, and the quantum confinement. Recently, advanced growth techniques have made it possible to fabricate high-quality semiconductor rings, ${ }^{25}$ which have attracted considerable attention due to intriguing quantum interference phenomenon arising from their unique topological geometry. ${ }^{26}$ The $\mathrm{AB}$ and the $\mathrm{AC}$ effects are typical examples of quantum interference, which have been demonstrated experimentally and theoretically on semiconductor rings. The interplay between the RSOI and DSOI results in a periodic potential in an isolated quantum ring that breaks the rotational symmetry, produces gaps in the energy spectrum and suppresses the persistent currents. ${ }^{27}$ This interesting feature leads to anisotropic spin transport and could be detected using the transport property in an opened two-terminal quantum ring. ${ }^{28,29}$

Current experimental state-of-the-art techniques make it possible to control the exact number of electrons in microstructures, for instance, by using finite-bias Coulombblockade and gate-voltage scans. ${ }^{30,31}$ In this paper, we investigate theoretically the two electron state in quantum rings under a perpendicular magnetic field in the presence of both RSOI and DSOI including the Coulomb interaction between the two electrons. We find that the single quantum ring behaves like a laterally coupled quantum dot, and the coupling strength induced by the interplay between the RSOI and DSOI can be tuned by changing the strengths of the SOIs, i.e., by an external gate. The interplay can lead to singlettriplet state mixing and anticrossing behavior between the singlet and triplet states with changing magnetic field. Interestingly, we find here that the two electron ground state displays a spatial anisotropic distribution in the quantum ring, a bar-bell-like ground state along the specific crystallographic direction, i.e., [110] or [1 10$]$, which can be switched rapidly by reversing the direction of the perpendicular electric field. This anisotropic distribution results in anisotropic optical properties that provide us with a possible way to detect it experimentally. The ground state also exhibits a singlettriplet transition at a critical magnetic field which can be tuned by changing the strength of the RSOI and DSOI.

The paper is organized as follows. In Sec. II the theoretical model is presented. The numerical results and discus- 


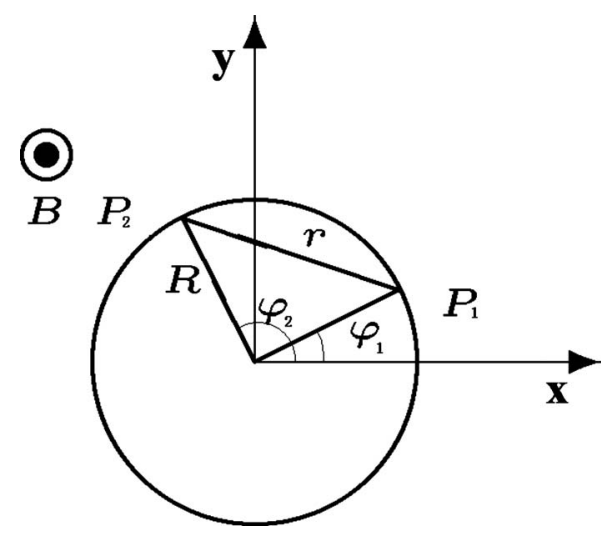

FIG. 1. Schematic of a one-dimensional quantum ring. A magnetic field is applied perpendicularly to the ring plane. The angles $\varphi_{1}$ and $\varphi_{2}$ denote the polar angles of the two electrons, respectively. $r$ is the distance between the two electrons.

sions are given in Sec. III. Finally, we give a brief conclusion in Sec. IV.

\section{THEORETICAL MODEL}

We consider a quantum ring structure schematically shown in Fig. 1. In the presence of both RSOI and DSOI, the Hamiltonian for two electrons in a finite-width quantum ring under a perpendicular magnetic field including the Coulomb interaction is

$$
\begin{aligned}
H= & \sum_{i=1}^{2}\left[\frac{\Pi_{i}^{2}}{2 m^{*}}+V_{\text {conf }}\left(r_{i}\right)+\frac{1}{2} g^{*} \mu_{B} B \sigma_{z}\right] \\
& +\sum_{i=1}^{2} \frac{\alpha}{\hbar}\left(\sigma_{x} \Pi_{i, y}-\sigma_{y} \Pi_{i, x}\right)+\sum_{i=1}^{2} \frac{\beta}{\hbar}\left(\sigma_{x} \Pi_{i, x}-\sigma_{y} \Pi_{i, y}\right) \\
& +\frac{e^{2}}{4 \pi \varepsilon_{0} \varepsilon\left|\mathbf{r}_{1}-\mathbf{r}_{2}\right|},
\end{aligned}
$$

where the first summation is the single-electron Hamiltonian in a ring under a perpendicular magnetic field. The vector potential $\mathbf{A}$ is taken in the symmetric gauge: $\mathbf{A}=B / 2(-y, x, 0)$, where the magnetic field $B$ is applied perpendicularly to the plane of the quantum ring. $m^{*}$ is the electron effective mass in units of the free-electron mass $m_{0}$. The Zeeman splitting is determined by the Bohr magneton $\mu_{B}=e \hbar / 2 m_{0}$ and the effective $g$-factor $g^{*} . \sigma_{i}(i=x, y, z)$ are the Pauli matrices. The second and third sum describes the RSOI and DSOI in the ring, respectively. The transverse confining potential is taken such that the electron motion is strictly one dimensional (1D), i.e., the electrons cannot move radially,

$$
V_{\text {conf }}(r)=\left\{\begin{array}{cc}
0, & r=R \\
\infty, & \text { otherwise. }
\end{array}\right.
$$

The difference among different confining potentials, e.g., the hard-wall and parabolic confining potentials, may lead to different quantitative features in the energy spectrum, but the important physics we are interested in is not affected by the precise form of the confining potential. The last term in Eq. (1) describes the Coulomb interaction between the two electrons in the ring. $|e|$ is the charge of the electron, $\varepsilon_{0}$ and $\varepsilon$ are the dielectric constant of vacuum and the semiconductor, respectively.

For a 1D ring in the presence of RSOI or DSOI we are not allowed to simply disregard all the terms proportional to derivatives with respect to $r$ in Eq. (1). This would lead to a non-Hermitian Hamiltonian. ${ }^{32}$ In this paper we take

$$
\begin{aligned}
H_{e}= & {\left[-i \frac{\partial}{\partial \varphi}+\frac{\Phi}{\Phi_{0}}+\frac{\bar{\alpha}}{2} \sigma_{r}-\frac{\bar{\beta}}{2} \sigma_{\varphi}(-\varphi)\right]^{2}-\frac{\bar{\alpha}^{2}+\bar{\beta}^{2}}{4} } \\
& +\frac{\bar{\alpha} \bar{\beta}}{2} \sin 2 \varphi+\frac{1}{2} \bar{g} b \sigma_{z}
\end{aligned}
$$

as our single-electron Hamiltonian, where $\sigma_{r}$ $=\cos \varphi \sigma_{x}+\sin \varphi \sigma_{y}, \sigma_{\varphi}=\cos \varphi \sigma_{y}-\sin \varphi \sigma_{x}, \Phi=B \pi a^{2}$ is the magnetic flux threading the ring, $\Phi_{0}=h / e$ is the flux unit, $\bar{\alpha}(\bar{\beta})=\alpha(\beta) / E_{0} R$ specifies the dimensionless RSOI (DSOI) strength, and $\bar{g}=g^{*} m^{*} / 2 m_{0}$ is the dimensionless $g$ factor. ${ }^{27}$

The total Hamiltonian can be written as

$$
H=\sum_{\alpha} E_{\alpha} a_{\alpha}^{+} a_{\alpha}+\frac{1}{2} \gamma \sum_{\alpha \beta \alpha^{\prime} \beta^{\prime}}\left\langle\alpha \beta|U| \alpha^{\prime} \beta^{\prime}\right\rangle a_{\alpha}^{+} a_{\beta}^{+} a_{\alpha^{\prime}} a_{\beta^{\prime}},
$$

where $E_{\alpha}$ is the energy of the $\alpha$ th single-electron level, which can be obtained numerically by solving the singleelectron Schrödinger equation for a ring in the presence of the SOIs [shown in Eq. (3)]. The parameter $\gamma=e^{2} / 4 \pi \varepsilon_{0} \varepsilon$ and $U=1 / r$. We adopt the configuration-interaction (CI) method to calculate the eigenvalues and eigenstates of the above Hamiltonian. The total wave function can be expanded $|\chi\rangle=\sum_{i} C_{i}|I\rangle$, where the state vector $|I\rangle=\left|\cdots 01_{i} 0 \cdots 01_{j} 0 \cdots\right\rangle$ $=a_{i}^{+} a_{j}^{+}|0\rangle$ with $i<j$. Here $|0\rangle$ represents the vacuum state and $a_{i}^{+}\left(a_{i}\right)$ is the electron creation (annihilation) operator of the states. $i$ and $j$ denoting the $i$ th and $j$ th single-electron energy levels, respectively. The matrix element of the total Hamiltonian can be calculated,

$$
\langle I|H| J\rangle=\left(E_{i}+E_{j}\right) \delta_{i p} \delta_{j q}+\gamma[\langle i j|U| q p\rangle-\langle i j|U| p q\rangle] .
$$

Solving this secular equation we get the eigenenergy and the eigenstate of the two electron system. The spin projection $\left\langle S_{z}\right\rangle=\left\langle\chi\left|S_{z}\right| \chi\right\rangle$ and total spin $\left\langle S^{2}\right\rangle=\left\langle\chi\left|S^{2}\right| \chi\right\rangle$ can be calculated numerically from the obtained eigenstate $|\chi\rangle$.

The optical-absorption rate is obtained within the electric dipole approximation,

$$
\begin{aligned}
W_{a b} & =\frac{2 \pi}{\hbar} \sum_{f}\left|\left\langle f\left|H_{e p}\right| i\right\rangle\right|^{2} \delta\left(E_{f}-E_{i}\right) \\
& =\frac{2 \pi}{\hbar} \sum_{f, i}\left(\frac{e A_{0}}{m^{*}}\right)^{2}\left|\left\langle f\left|\overrightarrow{\epsilon_{\lambda}} \cdot\left(\overrightarrow{p_{1}}+\overrightarrow{p_{2}}\right)\right| i\right\rangle\right|^{2} \times \delta\left(E_{f}-E_{i}-\hbar \omega\right),
\end{aligned}
$$

where $E_{i}$ and $E_{f}$ represent the energies of the initial and final states. $H_{e p}=\left(e / m^{*}\right)\left(\vec{A} \cdot \overrightarrow{p_{1}}+\vec{A} \cdot \vec{p}_{2}\right)$, where $A_{0}, \omega$, and $\epsilon_{\lambda}$ are the amplitude, frequency, and polarization vector of the incident linear-polarized light, and $\vec{p}_{1,2}$ are the canonical momenta of 


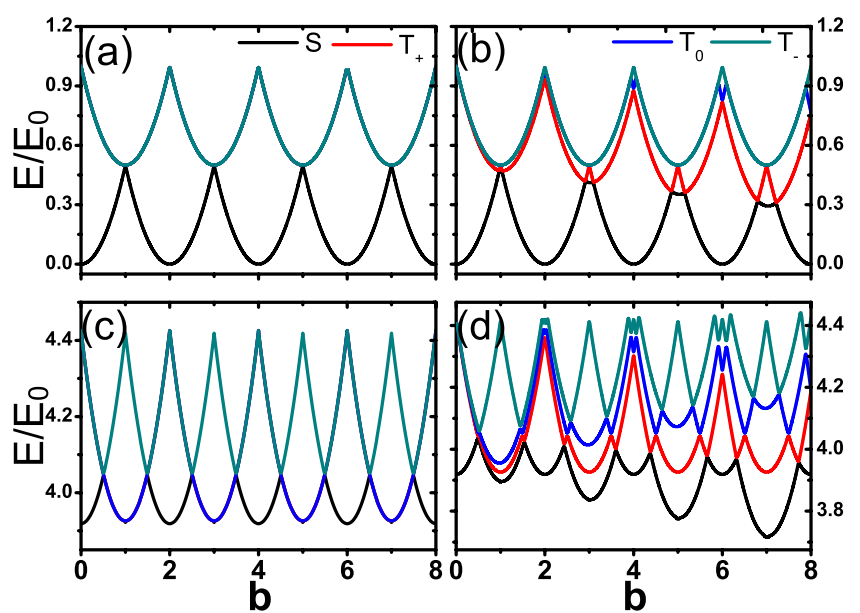

FIG. 2. (Color online) The energy spectrum for 1D GaAs rings without SOIs. (a) Without Coulomb interaction and Zeeman effect, (b) without Coulomb interaction but with Zeeman effect, (c) with Coulomb interaction but without Zeeman effect, and (d) with both Coulomb interaction and Zeeman effect.

the electrons. In the calculation we use a Lorentz broadening function $\Gamma / \pi /\left[\left(\hbar \omega-E_{12}\right)^{2}+\Gamma^{2}\right]$ for the energy delta function $\delta\left(E_{12}-\hbar \omega\right)$ and $\Gamma$ is the broadening parameter describing the homogeneous broadening of the energy levels in the ring [see the Appendix].

The accuracy of the calculated two electron energy spectrum depends on the number of possible many-particle configurations that are used, which is determined by the number of electrons $\left(N_{e}=2\right.$ in this paper), and the number of singleparticle states $N_{S}$. In the calculation we include 12 singleparticle electron states to ensure that the lower two electron states are accurate, e.g., the lowest four energy levels are accurate to $2.0 \times 10^{-4} \mathrm{meV}$. For simplicity, all physical quantities are taken dimensionless, e.g., the length unit is the radius of the ring $R$, the energy unit $E_{0}=\hbar^{2} / 2 m^{*} R^{2}$, and the magnetic field $b=e \hbar B / 2 m^{*} E_{0}$. The relevant parameters for GaAs are: ${ }^{33}$ the electron effective mass $m^{*}=0.067 m_{0}$, the effective $g$-factor $g^{*}=-0.44$, and the dielectric constant $\varepsilon=12.5$. For example, for $R=30 \mathrm{~nm}$ we find $E_{0}=0.633 \mathrm{meV}$ and $b=1.365$, when $B=1 \mathrm{~T}$.

\section{NUMERICAL RESULTS AND DISCUSSIONS}

In Fig. 2, we plot the energy spectra of the two electron system for different situations in the absence of the SOIs. From Fig. 2 one can find that the ground state is the singlet state $|S\rangle=(|\uparrow \downarrow\rangle-|\downarrow \uparrow\rangle) / \sqrt{2}$ (the total spin $S=0$ ) in zero magnetic field, formed by the two electrons occupying the lowest orbital with antiparallel spin. The first excited state is the spin triplet state $|T\rangle$ (total spin $S=1$ ), where the Pauli principle requires one electron to occupy a higher orbital state. The Zeeman effect leads to the splitting of the triplet state $\left|T_{0, \pm}\right\rangle$ [compare Figs. 2(a) and 2(b)], which increases with increasing magnetic field since the $z$ components of the total spin of the triplet states are different: $S_{z}=+1$ for $\left|T_{+}\right\rangle=|\uparrow \uparrow\rangle$, $S_{z}=0$ for $\left|T_{0}\right\rangle=(|\uparrow \downarrow\rangle+|\downarrow \uparrow\rangle) / \sqrt{2}$, and $S_{z}=-1$ for $\left|T_{-}\right\rangle=|\downarrow \downarrow\rangle$. A singlet-triplet ground-state transition takes place with in-
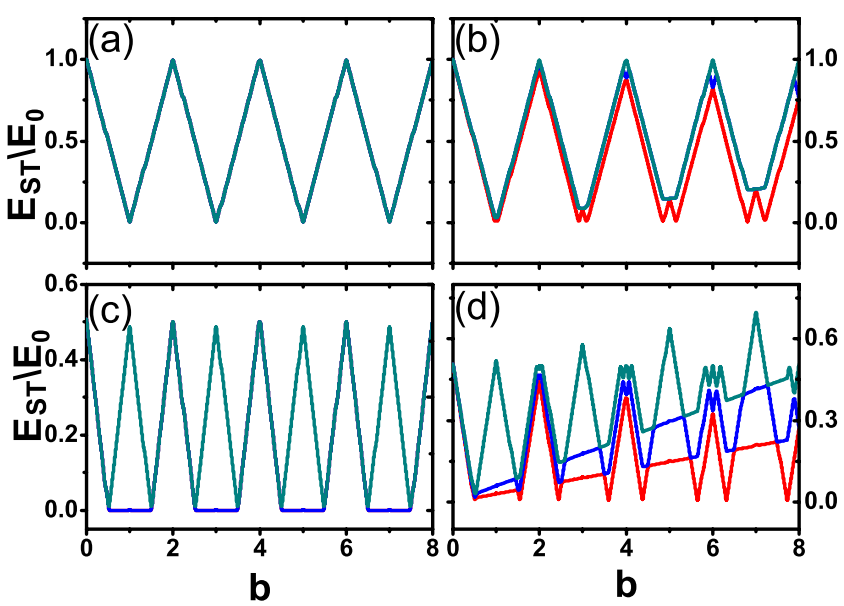

FIG. 3. (Color online) The same as Fig. 2 but for the energy difference between the singlet and triplet states $\Delta E_{S T}=E_{T_{0,+}}-E_{S}$ as a function of magnetic field.

creasing magnetic field. The Zeeman splitting results in different transition points of the ground state between the singlet state $S(S=0)$ and the triplet states $T_{0, \pm}$. The Coulomb interaction changes the energies of the singlet and triplet states [see Figs. 2(c) and 2(d)]. This feature leads to a significant lowering of the critical magnetic field $B_{c}$ determining the ground-state transition. The ground-state transition is a quantum phase transition driven by the magnetic field that reduces the energy difference between the singlet and triplet states, enhances the Coulomb repulsion interaction for the spin singlet state because the two electrons in the singlet state occupy the same orbital state while the two electrons in the triplet states occupy two different orbital states.

In Fig. 3, we plot the singlet-triplet splitting $E_{S T}$ as a function of magnetic field for various situations. The splitting $E_{S T}$ are determined by two factors: the Coulomb exchange interaction and the Zeeman effect. The Zeeman effect leads to three different crossing points for different triplet states $S_{z}=0, \pm 1$ while the Coulomb exchange interaction results in the splitting of the triplet states $S_{z}=0$ and $S_{z}= \pm 1$. The interplay between Coulomb interaction and Zeeman effect leads to a complicated behavior for the splitting $E_{S T}$ [see Fig. 3(d)].

Figure 4 shows $\left\langle S^{2}\right\rangle$ as a function of magnetic field $B$. The sudden jumps of $\left\langle S^{2}\right\rangle$ between 0 and 2 correspond to the singlet-triplet ground-state transition. One can find that the Coulomb interaction lowers the critical magnetic field $B_{c}$ and broadens the range of the triplet ground states [see Figs. 4(a) and 4(c)]. This feature can be seen clearly from Fig. 4. This is because the spin degeneracy of the triplet states can be lifted by the Zeeman effect and the Coulomb exchange interaction.

Next, we turn to the effect of the SOIs. Notice that the energy spectrum is exactly the same but shows opposite spin orientation with separate RSOI or DSOI for the same strengths $\bar{\alpha}=\bar{\beta}$ since the Hamiltonian of the RSOI is mathematically equivalent to that of the DSOI through a unitary transformation. ${ }^{27}$ This feature results from the SU(2) symmetry of the Hamiltonian.

The Coulomb interaction can couple the singlet and triplet states, which leads to an anticrossing and opens a gap in the 

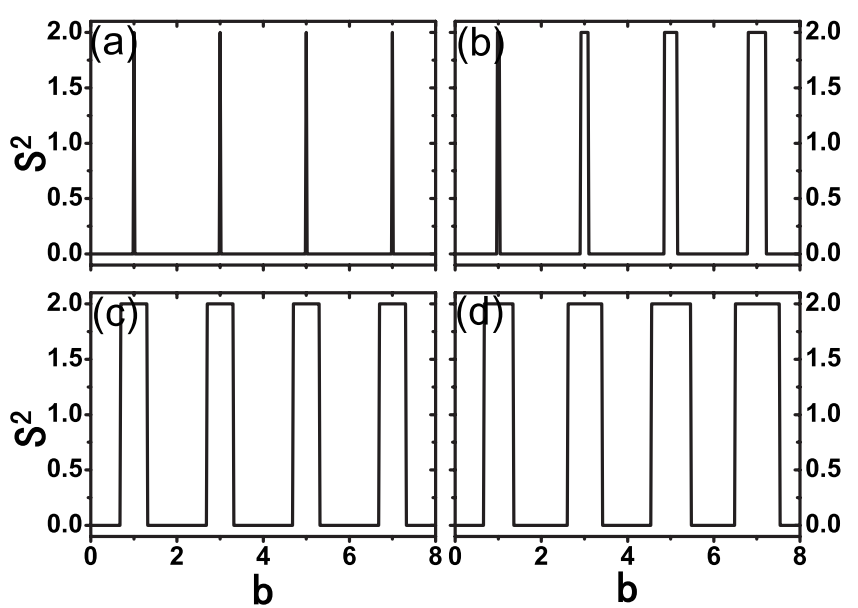

FIG. 4. $S^{2}$ for the ground state as a function of magnetic field $b$ without SOIs. (a) Without Coulomb interaction and Zeeman effect, (b) without Coulomb interaction but with Zeeman effect, (c) with Coulomb interaction but without Zeeman effect, and (d) with both Coulomb interaction and Zeeman effect.

energy spectrum. In the vicinity of the anticrossing points there is a strong mixing between the singlet-triplet states [see Figs. 5(c), 5(d), 6(c), and 6(d)].

The interplay between the RSOI and DSOI breaks the rotational symmetry and results in an azimuthal periodic potential, consequently it leads to an azimuthal anisotropic electron distribution. The electron distribution of the ground state shows a bar-bell-like shape along the specific crystallographic directions $\varphi= \pm \pi / 4$. The Coulomb repulsion makes the two electrons tend to avoid each other and localize at the two opposite sides but at any crystallographic direction. The broken rotational symmetry makes two electron localize along the crystallographic directions $\varphi= \pm \pi / 4$, thus behaving like a laterally coupled quantum dot. This feature can be seen clearly from the two electron distribution of the ground state [see Figs. 7(a) and 7(c)]. Interestingly, one can switch the minima of the azimuthal periodic potential from [110] to
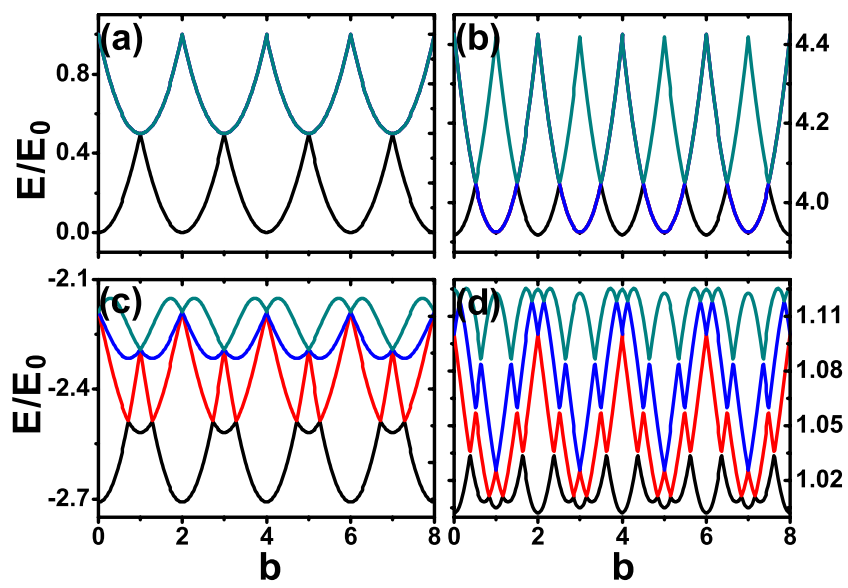

FIG. 5. (Color online) Energy spectrum of a 1D ring. (a) Without Coulomb interaction and SOIs, (b) with Coulomb interaction but without SOIs, (c) without Coulomb interaction but with SOIs, and (d) with both Coulomb interaction and SOIs. $\bar{\alpha}=2.0, \bar{\beta}=1.0$.
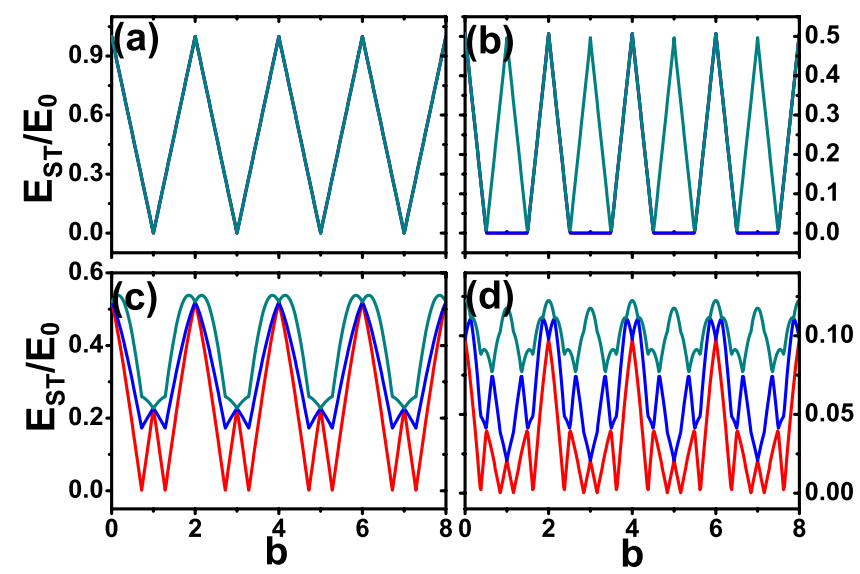

FIG. 6. (Color online) The energy differences between the single and triplet states $\Delta E_{S T}=E_{T_{0,+}}-E_{S}$ as a function of magnetic field. (a) Without Coulomb interaction and SOIs, (b) with Coulomb interaction but without SOIs, (c) without Coulomb interaction but with SOIs, and (d) with both Coulomb interaction and SOIs. $\bar{\alpha}=2.0, \bar{\beta}=1.0$.

$[1 \overline{1} 0]$ rapidly by reversing the direction of the perpendicular electric field, i.e., $\bar{\alpha}$ to $-\bar{\alpha}$. The orientation of the bar-belllike ground state distribution can be switched from [110] to [1̄ㅣ] [see Figs. 7(b) and 7(d)].

Figures 8(a) and 8(b) shows the phase diagrams of the two electron system as function of the magnetic field and the strength of the SOIs. One can see that the singlet-triplet ground-state transition depends sensitively on the relative strengths of the RSOI and DSOI which can be seen clearly from Fig. 8(a) $(\bar{\alpha}=\bar{\beta})$ (Ref. 34) and Fig. 8(b) $(\bar{\alpha}=2 \bar{\beta})$. This
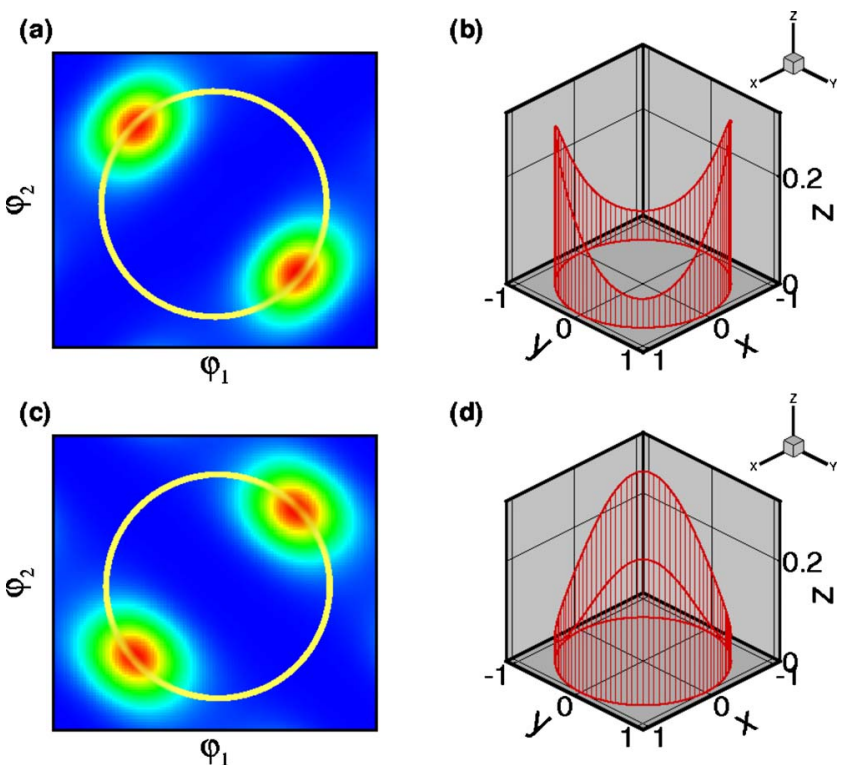

(d)

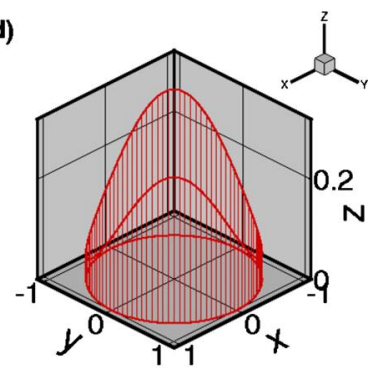

FIG. 7. (Color online) [(a) and (c)] Contour plot and [(b) and (d)] three-dimensional plot of the spatial distribution of the ground state of the two electron ring as a function of the azimuthal angles of electron 1 and 2 when including the Coulomb interaction. [(a) and (b)] $\bar{\alpha}=2, \bar{\beta}=1$ and [(c) and (d) $] \bar{\alpha}=-2, \bar{\beta}=1$. The solid yellow circle in (a) and (c) indicates the quantum ring. 
(a)

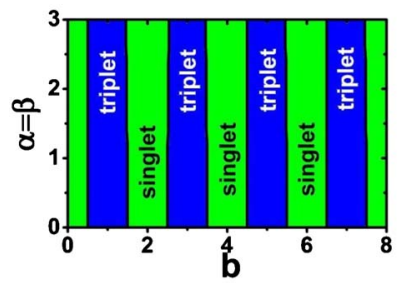

(b)

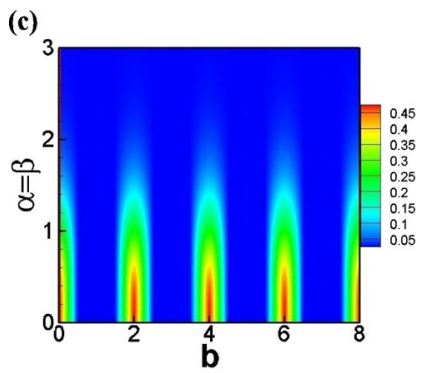

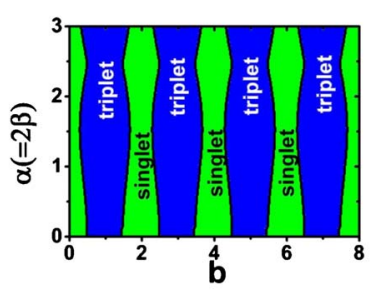

(d)

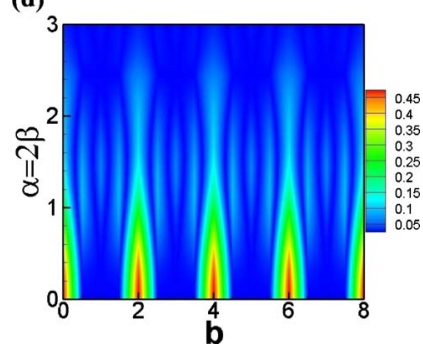

FIG. 8. (Color online) Phase diagrams as function of magnetic field and the strength of the SOIs for (a) $\bar{\alpha}=\bar{\beta}$ and (b) $\bar{\alpha}=2 \bar{\beta}$. [(c) and (d)] Contour plots of the energy differences between the singlet and triplet states $\Delta E_{S T}$ for (c) $\bar{\alpha}=\bar{\beta}$ and (d) $\bar{\alpha}=2 \bar{\beta}$.

character can be understood from the behavior of the singlettriplet splitting $\Delta E_{S T}$ [see Figs. $8(\mathrm{c})$ and $\left.8(\mathrm{~d})\right]$ where the exchange interaction is very sensitive to the spin orientation of the two electrons. The latter can change heavily even by a slight variation in the RSOI and DSOI. ${ }^{27}$ Therefore the relative strength between the RSOI and DSOI would lead to a different behavior of the singlet-triplet splitting $\Delta E_{S T}$, which results in different phase diagrams.

Finally we turn to the discussion how to detect the two electron spatial anisotropic distribution. The two electron spin states have been previously measured by the tunneling process through quantum dots. ${ }^{35}$ Here we propose an optical method, i.e., optical absorption in the infrared regime, to detect the electron spatial anisotropic distribution. This method is able to detect more directly the anisotropic electron distribution and the overlap factor between the ground and first excited states, i.e., anisotropic absorption. Consider a beam of linear-polarized light incident along the $z$ axis. We calculate the optical-absorption index in the infrared regime. In Fig. 9 we plot the optical absorption index as a function of the angle $\theta$ of the polarization plane of the incident linearpolarized light with respect to the $x$ axis. From the figure, one can find that the optical-absorption index oscillates periodically with increasing crystallographic angle $\theta$. Due to the azimuthal periodic potential induced by the interplay between the RSOI and DSOI and the Coulomb repulsion between the two electrons the electron distributions are strongly localized in the ring along the crystallographic direction $\varphi= \pm \pi / 4$, i.e., a bar-bell-like distribution (see Fig. 7). The bar-bell-like distribution certainly leads to an anisotropic behavior of the optical absorption, i.e., periodical oscillation of the optical absorption as a function of the crystallographic direction $\theta$ (see Fig. 9). By reversing the direction of the perpendicular electric field, i.e., $\bar{\alpha}$ to $-\bar{\alpha}$, the optical absorption will change strongly since the electric dipole of the two electrons change from parallel to perpendicu-

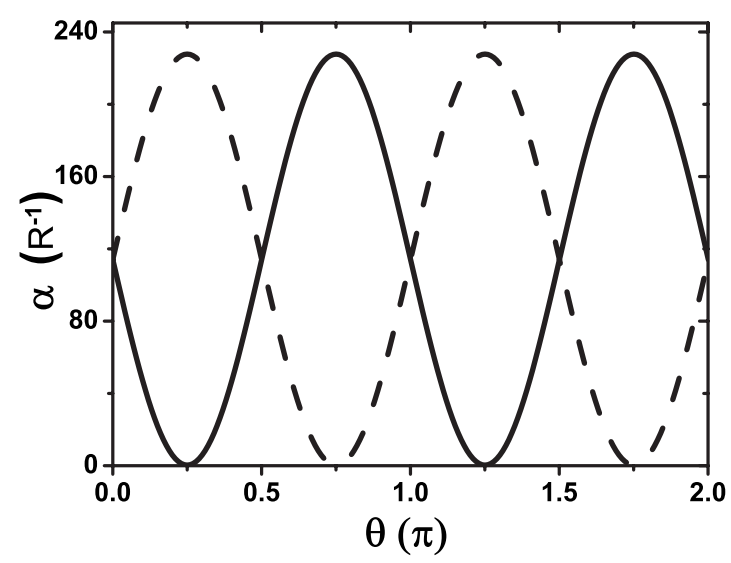

FIG. 9. The optical-absorption coefficient as a function of the direction of the polarized vector of the incident linear-polarized light. $\bar{\beta}=9, \Gamma=1 / \pi^{2}, \omega=3.35 E_{0}$ (the solid line) $\bar{\alpha}=10$ and (the dashed line) $\bar{\alpha}=-10$.

lar with respect to the polarization vector of the incident light. This large variation provides us with an efficient tool to detect the anisotropy in the electron distribution.

\section{SUMMARY}

We investigated theoretically the two electron states in quantum rings in the presence of SOIs under a perpendicular magnetic field using the CI method. We found that the single quantum ring behaves like a laterally coupled quantum dot, and the coupling strength induced by the interplay between the RSOI and DSOI can be tuned by changing the strength of the SOIs. The interplay can lead to singlet-triplet state mixing and to an anticrossing behavior between the singlet and triplet states with changing magnetic field. The Coulomb interaction can lead to a decrease (increase) in the energy of the triplet (singlet) state, consequently it reduces significantly the critical magnetic field corresponding to the singlet-triplet transition. Interestingly, the two electron ground state displays a bar-bell-like spatial anisotropic distribution in the quantum ring fixed at a specific crystallographic direction, which results in anisotropic optical properties that can be tuned electrically.

\section{ACKNOWLEDGMENTS}

This work was supported by NSFC under Grants No. 60525405 and No. 10874175 and the Belgium Science Policy (IAP).

\section{APPENDIX: OPTICAL-ABSORPTION INDEX}

When a beam of light is incident on a 1D ring, the system Hamiltonian reads

$$
H=\frac{1}{2 m^{*}}\left(\vec{p}_{1}+e \vec{A}\right)^{2}+\frac{1}{2 m^{*}}\left(\vec{p}_{2}+e \vec{A}\right)^{2}+H_{\mathrm{SO}}+V+H_{\text {Coul }},
$$

where the spin-orbit term $H_{\mathrm{SO}}=\alpha\left(\sigma_{x} k_{y}-\sigma_{y} k_{x}\right)$ $+\beta\left(\sigma_{x} k_{x}-\sigma_{y} k_{y}\right)$, the Coulomb interaction term 
$H_{C o u l}=\frac{1}{4 \pi \varepsilon_{0} \varepsilon} \frac{e^{2}}{r}$, the radial confining potential $V$, and the vector potential $\vec{A}$ of incident light,

$$
\vec{A}=\sum_{k} A_{0} \vec{\epsilon}_{\lambda}\left\{a_{k \lambda} e^{i\left(-\omega t+k_{\lambda} \cdot \vec{r}\right)}+a_{k \lambda}^{+} e^{i\left(\omega t-\overrightarrow{k_{\lambda}} \cdot \vec{r}\right)}\right\}
$$

here $a_{k \lambda}, a_{k \lambda}^{+}$are photon annihilation and creation operators, respectively. $k_{\lambda}$ is the wave vector of the incident light. The first and second terms in Eq. (A2) describe the photon absorption and emission processes, respectively.

Adopting the electric dipole approximation, the electronphoton interaction term becomes

$$
H_{e p}=\frac{e}{m^{*}}\left(\vec{A} \cdot \vec{p}_{1}+\vec{A} \cdot \vec{p}_{2}\right)
$$

and the absorption rate is

$$
W_{a b}=\frac{2 \pi}{\hbar} \sum_{\lambda, f}\left|\left\langle f\left|H_{e p}\right| i\right\rangle\right|^{2} \delta\left(E_{f \lambda}-E_{i \lambda}\right),
$$

where $|i\rangle$ and $|f\rangle$ represent the initial and final states which are the direct product of the electron states and the photon states $\left(\Pi\left|n_{\lambda}\right\rangle\right) . E_{i \lambda}$ and $E_{f \lambda}$ represent the total energy that are the sum of the electron and photon energy of the initial and final states. The absorption and emission rates can be written as

$$
\begin{aligned}
W_{a b}= & \frac{2 \pi}{\hbar} \sum_{\lambda, f, i}\left(\frac{e A_{0}}{m^{*}}\right)^{2}\left|\left\langle f\left|\overrightarrow{\epsilon_{\lambda}} \cdot\left(\vec{p}_{1}+\vec{p}_{2}\right)\right| i\right\rangle\right|^{2} \\
& \times n_{\lambda} \delta\left(E_{f}-E_{i}-\hbar \omega\right), \\
W_{e m}= & \frac{2 \pi}{\hbar} \sum_{\lambda, f, i}\left(\frac{e A_{0}}{m^{*}}\right)^{2}\left|\left\langle i\left|\overrightarrow{\epsilon_{\lambda}} \cdot\left(\vec{p}_{1}+\vec{p}_{2}\right)\right| f\right\rangle\right|^{2} \\
& \times\left(n_{\lambda}+1\right) \delta\left(E_{i}-E_{f}+\hbar \omega\right),
\end{aligned}
$$

and the total rate is given by

$$
W_{\text {stim }}=W_{a b}+W_{e m} .
$$

In the $1 \mathrm{D}$ ring system the emitted photons are directed in arbitrary directions in space and the number of photons reaching the detector is negligible. The total rate is

$$
W_{\text {stim }}=\frac{2 \pi}{\hbar}\left(\frac{e A_{0}}{m^{*}}\right)^{2} \sum_{2,1}\left|\left\langle 2\left|\overrightarrow{\epsilon_{\lambda}} \cdot\left(\vec{p}_{1}+\vec{p}_{2}\right)\right| 1\right\rangle\right|^{2} n_{\lambda} \delta\left(E_{12}-\hbar \omega\right),
$$

where $E_{12}=E_{2}-E_{1}$.

Light damping in media can be described as

$$
F(x)=F_{0} e^{-\alpha x}
$$

where $\alpha$ is the absorption index of the medium and $F_{0}$ is the flux at $x=0$.

The energy flux equation is

$$
\nabla \cdot \vec{F}+\frac{\partial n_{\lambda}}{\partial t}=0
$$

and the flux can also be written as $F=v_{g} n_{\lambda}$, here $v_{g}=c / \eta$ is the speed of energy flux and $\eta$ is the refraction index of the medium. Thus

$$
\alpha=\frac{1}{F} \frac{d n_{\lambda}}{d t}=\frac{W_{\text {stim }}}{v_{g} n_{\lambda}}
$$

and from Eq. (A8) we find

$$
\alpha=\frac{2 \pi}{\hbar}\left(\frac{e A_{0}}{m^{*}}\right)^{2} \sum_{2,1} \frac{\left|\left\langle 2\left|\overrightarrow{\epsilon_{\lambda}} \cdot\left(\vec{p}_{1}+\vec{p}_{2}\right)\right| 1\right\rangle\right|^{2}}{c / \eta} \delta\left(E_{12}-\hbar \omega\right) .
$$

\footnotetext{
*kchang@semi.ac.cn

${ }^{1}$ S. A. Wolf, D. D. Awschalom, R. A. Buhrman, J. M. Daughton, S. von Molnár, M. L. Roukes, A. Y. Chtchelkanova, and D. M. Treger, Science 294, 1488 (2001).

${ }^{2}$ T. Chakraborty and P. Pietiläinen, Phys. Rev. B 71, 113305 (2005).

${ }^{3}$ P. Pietiläinen and T. Chakraborty, Phys. Rev. B 73, 155315 (2006).

${ }^{4}$ M. Ciorga, A. Wensauer, M. Pioro-Ladriere, M. Korkusinski, J. Kyriakidis, A. S. Sachrajda, and P. Hawrylak, Phys. Rev. Lett. 88, 256804 (2002).

${ }^{5}$ D. Grundler, Phys. Rev. Lett. 84, 6074 (2000).

${ }^{6}$ J. Nitta, T. Akazaki, H. Takayanagi, and T. Enoki, Phys. Rev. Lett. 78, 1335 (1997).

${ }^{7}$ J. Nitta, F. E. Meijer, and H. Takayanagi, Appl. Phys. Lett. 75, 695 (1999).

${ }^{8}$ M. König, A. Tschetschetkin, E. M. Hankiewicz, J. Sinova, V. Hock, V. Daumer, M. Schäfer, C. R. Becker, H. Buhmann, and
}

L. W. Molenkamp, Phys. Rev. Lett. 96, 076804 (2006).

${ }^{9}$ I. Žutić, J. Fabian, and S. D. Sarma, Rev. Mod. Phys. 76, 323 (2004).

${ }^{10}$ R. Winkler, Physica E 22, 450 (2004).

${ }^{11}$ S. Datta and B. Das, Appl. Phys. Lett. 56, 665 (1990).

${ }^{12}$ E. I. Rashba, Sov. Phys. Solid State 2, 1109 (1960).

${ }^{13}$ Y. A. Bychkov and E. I. Rashba, J. Phys. C 17, 6039 (1984).

${ }^{14}$ G. Dresselhaus, Phys. Rev. 100, 580 (1955).

${ }^{15}$ D. Bhowmik and S. Bandyopadhyay, Physica E 41, 587 (2009).

${ }^{16}$ S. Lamari, Phys. Rev. B 67, 165329 (2003).

${ }^{17}$ J. Nitta, T. Bergsten, Y. Kunihashi, and M. Kohda, J. Appl. Phys. 105, 122402 (2009).

${ }^{18}$ S. M. Frolov, S. Lüscher, W. Yu, Y. Ren, J. A. Folk, and W. Wegscheider, Nature (London) 458, 868 (2009).

${ }^{19}$ M. Studer, S. Schön, K. Ensslin, and G. Salis, Phys. Rev. B 79, 045302 (2009).

${ }^{20}$ T. Kaneko, M. Koshino, and T. Ando, Phys. Rev. B 78, 245303 (2008). 
${ }^{21}$ Y. Li and Y. Q. Li, Phys. Rev. B 78, 195325 (2008).

${ }^{22}$ B. Molnár, F. M. Peeters, and P. Vasilopoulos, Phys. Rev. B 69, 155335 (2004).

${ }^{23}$ D. Frustaglia and K. Richter, Phys. Rev. B 69, 235310 (2004).

${ }^{24}$ M. Bayer, M. Korkusinski, P. Hawrylak, T. Gutbrod, M. Michel, and A. Forchel, Phys. Rev. Lett. 90, 186801 (2003).

${ }^{25}$ A. Fuhrer, S. Lüescher, T. Ihn, T. Heinzel, K. Ensslin, W. Wegscheider, and M. Bichler, Nature (London) 413, 822 (2001).

${ }^{26}$ S. Souma and B. K. Nikolic, Phys. Rev. Lett. 94, 106602 (2005).

${ }^{27}$ J. S. Sheng and K. Chang, Phys. Rev. B 74, 235315 (2006).

${ }^{28}$ M. Wang and K. Chang, Phys. Rev. B 77, 125330 (2008).

${ }^{29}$ M. Wang and K. Chang, Appl. Phys. Lett. 94, 052108 (2009).

${ }^{30}$ R. C. Ashoori, Nature (London) 379, 413 (1996).
${ }^{31}$ S. Tarucha, D. G. Austing, T. Honda, R. J. van der Hage, and L. P. Kouwenhoven, Phys. Rev. Lett. 77, 3613 (1996).

${ }^{32}$ F. E. Meijer, A. F. Morpurgo, and T. M. Klapwijk, Phys. Rev. B 66, 033107 (2002).

${ }^{33}$ Physics of Group IV Elements and III-V Compounds, LandoltBörnstein, New Series, Group III Vol. 17, edited by O. Madelung (Springer-Verlag, Berlin, 1982).

${ }^{34}$ J. Schliemann, J. C. Egues, and D. Loss, Phys. Rev. Lett. 90, 146801 (2003).

${ }^{35}$ R. Hanson, L. M. K. Vandersypen, L. H. Willems van Beveren, J. M. Elzerman, I. T. Vink, and L. P. Kouwenhoven, Phys. Rev. B 70, 241304(R) (2004). 